\title{
Rapid solidification of steels
}

\author{
P RAMACHANDRARAO \\ National Metallurgical Laboratory (CSIR), Jamshedpur 831007 , India
}

\begin{abstract}
Solidification at very high rates of cooling results in considerable refinement in the microstructure of alloys. It enables the formation of extremely fine grains, extends solid solubilities and leads to the nucleation of many metastable crystalline phases. We discuss briefly the methods of rapid solidification and their impact on microstructure and structure of alloys. Each of the aspects of structure development is illustrated with examples from steels.
\end{abstract}

Keywords. Rapid solidification; steels; microstructural refinement; metastable phases.

\section{Introduction}

The advent of rapid solidification over three decades ago has placed a new tool in the hands of alloy development engineers. The phenomenal rates of cooling, often in excess of $10^{6} \mathrm{Ks}^{-1}$, have altered the structure and microstructure of materials drastically. The product, together with judicious heat treatment, is capable of yielding a variety of properties to satisfy diverse engineering needs. The present paper attempts to briefly discuss some of the available techniques of rapid solidification and the impact of rapid solidification on structure. It illustrates various aspects of the problem by choosing examples from steels and related alloys.

\section{Rapid solidification techniques}

Currently available rapid solidification techniques yield a variety of shapes. For example, sheet-like specimen can be produced by twin roll, twin belt, planar flow casting and other similar techniques while a ribbon is produced by melt spinning or other single roll techniques. Wire-like samples are made by spinning in water and melt extraction. Irregular perforated foils can be made by the gun technique while a large number of atomization techniques yield powders. Besides these, we also have techniques like laser surface glazing, electron beam melting etc in which a thin surface layer of a solid is taken into the molten state and gets quenched on switching off the heating source. Table 1 summarizes the rates of cooling attained in some of these methods. Of all the methods available now, melt spinning is by far the most used. Some of the important aspects of cooling during melt spinning are described below.

\section{Cooling rates in melt spinning}

In melt spinning, a thin stream of liquid is ejected gently on to a wheel made of high conducting material. The liquid forms a pool and the centrifugal forces generated by the high speed rotation of the wheel drums the liquid out in the form of a ribbon. The partially frozen ribbon stays in contact with the liquid for a short time before leaving it. During the dwell time of the ribbon, heat is extracted from it by conduction into the wheel. 
Table 1. A comparison of cooling rates and heat transfer coefficients in different methods of rapid solidification.

\begin{tabular}{|c|c|c|c|}
\hline Method & $\begin{array}{l}\text { Atomization } \\
\text { with forced } \\
\text { convective } \\
\text { cooling }\end{array}$ & $\begin{array}{l}\text { Melt } \\
\text { spinning }\end{array}$ & $\begin{array}{l}\text { Surface } \\
\text { melting and } \\
\text { self-quenching }\end{array}$ \\
\hline Shape of product & Sphere & Ribbon & Thin surface layer \\
\hline $\begin{array}{c}\text { Heat transfer } \\
\text { coefficient } \\
\left(\mathrm{W} / \mathrm{m}^{2} \mathrm{~K}\right)\end{array}$ & $<10^{5}$ & $10^{6}$ & Tends to infinity \\
\hline $\begin{array}{l}\text { Maximum cooling } \\
\text { rate }(K / s)\end{array}$ & $<10^{6}$ & $10^{5}-10^{7}$ & $10^{8}$ \\
\hline
\end{tabular}

The temperature $(T)$ at a point on the ribbon varies as a function of time $(t)$ according to

$$
T=T_{w}+\left(T_{i}-T_{w}\right) \exp \left(-h t / x p c_{p}\right)
$$

where $T_{w}$ is the temperature of the wheel and $T_{i}$, the initial temperature of the ribbon (Kim and Cantor 1990). As can be seen from (1), $T$ is a function of the heat transfer coefficient $(h)$, thickness of ribbon formed $(x)$, density $(p)$ and heat capacity $(c)$ of the ribbon. Under Newtonian conditions of cooling, the average cooling rate experienced by the ribbon can be expressed by

$$
\mathrm{d} T / \mathrm{d} t=h\left(T_{i}-T_{w}\right) / x p c_{p} .
$$

For a given material, $x$ and $h$ control the cooling rate. $x$ itself is dependent on the volumetric flow rate of the melt from the nozzle $(Q)$ and wheel speed $(V)$ according to

$$
x^{2} V / Q^{1 / 2}=\text { constant. }
$$

By a judicious balance of volume flow rate and wheel speed various thicknesses of ribbon can be produced. The consequent cooling rates vary in the range of $10^{5}-$ $10^{7} \mathrm{Ks}^{-1}$.

\section{Consolidation of rapidly solidified alloys}

The rapidly solidified material is amenable to consolidation by some of the following routes: (i) hot extrusion, (ii) hot isostatic pressing (HIP), (iii) closed die forging and (iv) high energy compaction. For the purpose of extrusion, the material is coldpressed, canned and evacuated, and then hot-extruded. Reduction in areas in the ratio of up to $30: 1$ are possible. After extrusion the canning material is removed by machining.

HIP, in its current stage, enables the production of objects of up to $1.0 \mathrm{~m}$ in diam. and $2.0 \mathrm{~m}$ in length with gas pressures in the range of $100-200 \mathrm{MPa}$. In recent years, methods have also been developed to produce near-net shaped die cavities directly by HIP with the aid of ceramic core technology and rapidly solidified tool steel powders (Staski et al 1988). One of the major disadvantages of HIP method is its high capital 


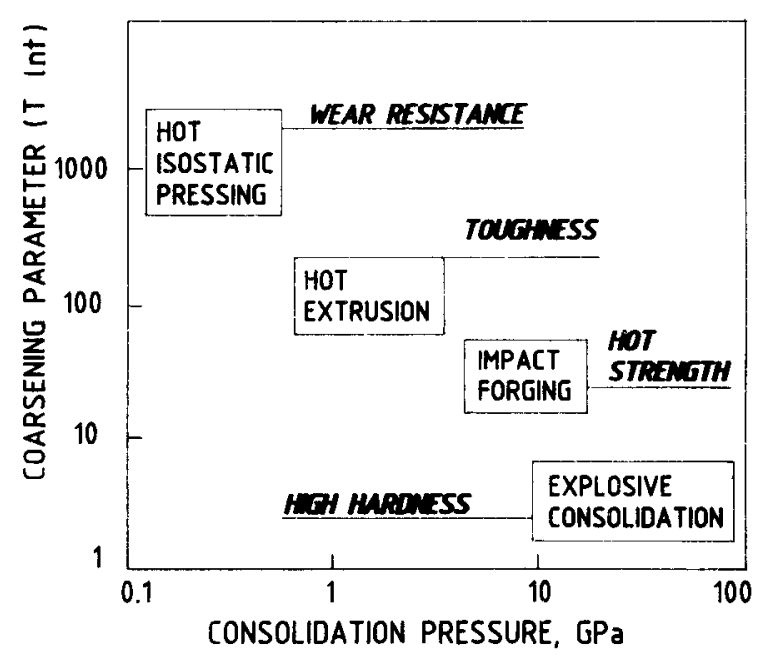

Figure 1. Range of the processes and operating parameters for the consolidation of rapidly solidified powders.

cost. Lately, pseudo-HIP processes such as rapid omnidirectional compaction (ROC), CERACON, STAMP and other similar processes which employ a granular medium for the transmission of pressure caused by conventional mechanical or hydraulic presses have emerged (Lawley 1985). For shapes which are non-cylindrical, the conventional hot closed-die forging has found favour. Rapidly solidified powders which are prone to decomposition and associated structural changes are best compacted by explosive or high velocity impact compaction techniques. Such a compaction can be carried out at room temperature with only the material making a brief excursion to the high temperature domain with surface melting.

Various methods available for consolidation can also be classified on the basis of extent of deformation to which the material is subjected. In processes such as HIP, there is none or little material deformation. In hot or cold forging, hot extrusion, pseudo-HIP etc. deformation is responsible for attaining the desired final densities. The specific properties attained as a result of compaction will depend upon the extent of deformation as well as structural and microstructural changes associated with it (Adam 1986). Figure 1 illustrates the changes in properties in terms of a coarsening parameter and the consolidation pressure. As can be seen, different methods are preferred for attaining each of the range of properties from wear resistance to hot strength.

\section{Effect of rapid solidification}

Materials science is concerned with the structure property correlations as well as the processing of materials for useful applications. Rapid solidification affects both these aspects. On the structural side it brings about some changes such as (a) refining of microstructural features, (b) reduction in segregation, (c) extension of solid solubility limits, and (d) production of novel metastable phases. Also it can drastically influence processing technologies by (a) simplification or elimination of hot rolling 
steps, (b) enabling the manufacture of otherwise difficult-to-hot roll materials, and (c) simplification of casting and rolling facilities.

\section{Refinement of microstructure}

The morphological and structural changes induced by rapid solidification of steels are (i) reduction in segregation, (ii) increase in the concentration of point defects, (iii) refinement of austeritic grain size, (iv) production of finer martensitic grain size, (v) formation of metastable solid solutions, and (vi) generation of metastable intermediate phases.

The extent of segregation observed after solidification depends critically on the morphology of growth and compositional changes at the solid-liquid interface. These in turn are functions of the rate of movement of the interface and rate of heat extraction. Whether or not compositional changes occur at the solid-liquid interface is dependent on thermodynamic and kinetic factors. A liquid can undergo diffusionless transformation to solid without solute partitioning only below a temperature $T_{0}^{L / \alpha}$. At this temperature the liquid $(L)$ and the solid $(\alpha)$ of the same composition have equal free energies. If a liquid can be undercooled to a temperature $T<T_{0}^{L / \alpha}$, it may solidify to give $\alpha$ without solute partitioning. A very high value of $T_{0}^{L / \alpha}$. is thus conducive to the formation of chemically homogeneous solid at small undercoolings. In such cases one can expect a high degree of supersaturation in solid solution phases with good compositional homogeneity. Such phases are produced by supercooling the liquid rapidly to $T<T_{0}^{L / \alpha}$ and/or preventing heterogeneous nucleation in the melt.

In case solute redistribution occurs at the interface, theoretical models show that the thermal gradient $\left(G_{L}\right)$ into the liquid at the solid-liquid interface and the rate of growth of the solid $(R)$ are important (Flemings 1974). These are related to each other through

$$
G_{L} / R=m_{L} C_{0}\left(k_{\text {eq }}-1\right) / D_{L} k_{\text {eq }}
$$

where $m_{L}$, slope of the liquidus line, $k_{\text {eq }}$, ratio of concentration of solid $\left(C_{S}\right)$ and liquid $\left(C_{L}\right)=C_{S} / C_{L}, D_{L}$, diffusivity of solute in the liquid and $C_{0}$, initial solute concentration in the liquid under consideration.

It can be demonstrated that a planar solid-liquid interface is stable only when $G_{L} / R$ exceeds a critical value given by the right hand side of (4). At lower values of $G_{L} / R$ the planar interface becomes morphologically unstable and degenerates to yield either cellular or dendritic structures as shown in figure 2.

Product of $G_{L}$ and $R$ has dimensions of cooling rate and is a measure of the same. Also shown in figure 3 are profiles which define different cooling rates. It can be seen that increasing the rate of cooling leads to smaller solidification times and shorter diffusion distances. Accordingly, increase in cooling rate tends to reduce the distances between microsegregated regions.

When the growth rate is increased significantly, the distance between the protrusions generated by morphological instability decrease. Under such conditions surface tension effects cannot be ignored. Further, latent heat is generated at the solid-liquid interface at a rapid rate and strongly influences the thermal field. Coriell and Sekerka (1980) analysed the stability of the interface taking these aspects into account. Under such conditions, figure 2 gets modified to yield one of the type of figure 3 . We note 


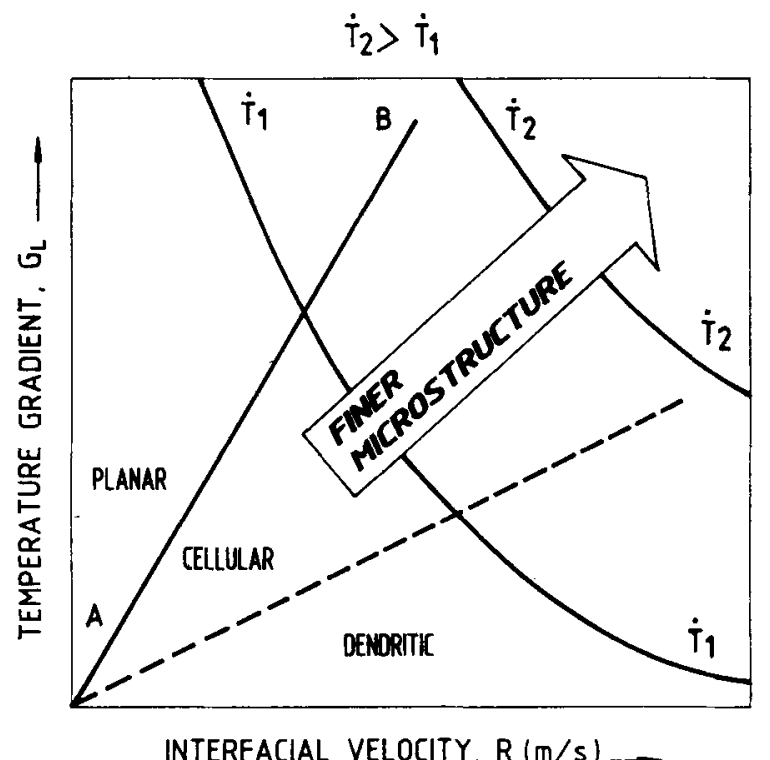

Figure 2. Predicted microstructure of solidification product and its dependence on temperature gradient and growth rate.

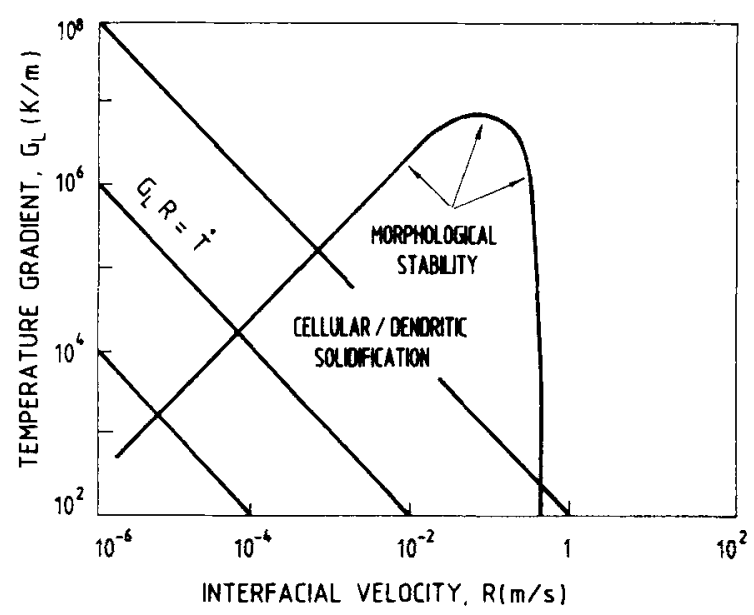

Figure 3. Predicted microstructures of rapidly solidified material and its dependence on temperature gradient and growth rate.

that plane front gets increasingly stabilized at higher growth rates as well. Such a stabilization does not necessarily preclude solute redistribution. It only suggests that the equilibrium distribution coefficient $\left(k_{e}\right)$ is no longer valid and that prevailing ratio $(k)$ of $C_{s} / C_{L}$ is greater than $k_{e}$. It can even become unity. Such a process is termed solute trapping. This is essentially a kinetic effect and many models are available to describe this phenomenon and derive expressions for $k$ in terms of $k_{\text {eq }}$ and growth rate. One such expression due to Aziz (1982) is

$$
k=\left(k_{\mathrm{eq}}+d\right) /(1+d)
$$


where $d=R a / D_{L}$ and is a dimensionless velocity, $a$, being the solute jump length across the interface. Very high values of $k$ are preferred for segregation free microstructures.

One aspect of importance in rapid solidification is the large undercooling observed. In $\mathrm{Fe}-\mathrm{Ni}$ alloys it can be as large as $300 \mathrm{~K}$ for cooling rates in the range $5 \times 10^{6}-$ $10^{7} \mathrm{Ks}^{-1}$ and $50-200 \mathrm{~K}$ at $5 \times 10^{5} \mathrm{Ks}^{-1}$ (Hayzelden et al 1983). Hence, diffusionless solidification is often observed in $\mathrm{Fe}-\mathrm{Ni}$ alloys. In other cases, initial portions to freeze exhibit diffusionless solidification while latter portions whose cooling rate is decreased due to evolution of latent heat show segregation. This yields $\approx 0.1 \mu \mathrm{m}$ cellular microstructure with nickel being segregated at the cell boundaries. The extent of segregation is, however, very small. By a careful photocalorimetric observation of rapid solidification, Cantor et al (1991) studied the freezing behaviour of $316 \mathrm{~L}$ type stainless steels. The observed temperature-time plots indicate that nucleation occurs at a temperature $T_{N}$ below the equilibrium solidus temperature $T_{s}$. The solute concentration of the solid so formed may vary and lie between that in the starting liquid $\left(C_{0}\right)$ and $C_{N}$ where $C_{N}$ is the solute concentration in the solid with the maximum driving force. The solid first spreads laterally to cover the entire surface of contact with the wheel. Columnar grains then grow. For the planar front to be stable the temperature to which the liquid recalesces $\left(T_{G}\right)$ should be less than $T_{s}$.

Cantor et al (1991) have also shown that a relationship exists between the average lateral grain diameter $d_{g}$ and the nucleation undercooling. $\Delta T_{N}\left(=T_{S}-T_{N}\right)$. It has the form

$$
\ln d_{g}=\text { constant }+\ln \Delta T_{N}+\left(Q / \Delta T_{N}^{2}\right)
$$

where $Q$ is an activation energy for nucleation. $\Delta T_{N}$ is in itself a function of the cooling rate. They also observed that

$$
\ln d_{g} \propto 1 /(\mathrm{d} T / \mathrm{d} t)^{2}
$$

for rapidly solidified $316 \mathrm{~L}$ stainless steel.

\section{Concentration of quenched-in defects}

The occurrence of point, line and planar defects in rapidly solidified materials has already been reviewed elsewhere (Anantharaman and Ramachandrarao 1973). Wood and Honeycombe (1974) studied the microstructure of rapidly solidified $\mathrm{Fe}-\mathrm{Cr}-\mathrm{Ni}$ austenitic steels and observed three types of structures viz. high-angle cellular, linear arrays of dislocation loops- and low-angle cellular arrays. The sequence follows highest to lowest cooling rates attained in a given foil. The dislocation loops observed at intermediate cooling rates lie in the $\langle 100\rangle$ direction and have Berger's vector of $(a / 2)\langle 110\rangle$. These are considered to be associated with vacancy coalescence and imply a high density of quenched-in vacancies.

Bae and Kelly (1988) have also studied defect microstructures in 304 stainless steel powders produced by centrifugal atomization (CA) and vacuum gas atomization (VGA) routes. They observed Frank type loops near the surface of helium containing CA powders. The formation of these loops is attributed to the condensation of vacancies on a close-packed plane at a helium bubble to reduce the pressure of the bubble. The interior regions of the powder showed stacking faults which may have evolved 
from Frank loops. In addition, a number of defects which could be attributed to noble gas atom-vacancy clusters have also been observed. There is also considerable evidence to suggest that such defects inhibit grain growth in parts obtained by consolidation of these powders.

\section{Grain refinement in rapidly solidified alloys}

Rapid solidification has yielded grain sizes as small as a few hundred Angströms (Ramachandrarao et al 1972). In most alloys grain diameters of the order of $0.01 \mu \mathrm{m}$ are attainable (Jones 1977). Many investigations have demonstrated the importance of fine grain size in reducing segregation, improving strength, ductility, fracture toughness and inducing super plasticity. There are two routes by which grain refinement can be achieved and they depend on either rapid heat extraction or on inducing large undercoolings. In the latter case, the extent of undercooling may be large enough to induce glass formation. Some of the finest microstructures obtained are a result of devitrification of an amorphous phase produced as an intermediate state. There is ample evidence to suggest that crystallization of the glassy phase occurs through homogeneous nucleation (Greer 1988). Grain refinement by rapid heat extraction, on the other hand, occurs by heterogeneous nucleation. The grain size $\left(d_{g}\right)$ will then depend on the heterogeneous nucleation frequency and prevalent growth rates. Under conditions where heat extraction is not a rate controlling step, Greer (1991) demonstrated that $d_{g}$ should vary inversely as the rate of cooling. This is in contrast to the arguments of Cantor et al (1991) who conclude that the columnar grain diameter should vary as per (6) above.

In $\mathrm{Fe}-\mathrm{Ni}$ alloys, the size of an austenite grain formed by conventional casting is of the order of $100 \mu \mathrm{m}$. By rapid solidification one can obtain grain sizes that are two orders of magnitude smaller (Inokuti and Cantor 1977, 1982). As a result of the fine austenitic grain size, the resultant martensite plate size is also very fine $(<1 \mu \mathrm{m})$. This reduction in size enhances the martensitic hardness considerably. Lowering of the austenitic grain size and increase of solubility of alloying elements in austenite due to rapid solidification alter the $M_{s}$. It has been observed that higher the cooling rate, lower is the $M_{s}$. This decrease in $M_{s}$ can increase the amount of retained austenite which softens the material and the overall hardness of the quenched alloy may be lowered. Inokuti and Cantor (1982) found a rapid fall in the hardness of $\mathrm{Fe}-\mathrm{Ni}$ alloys with $>20 \% \mathrm{Ni}$.

The reduction in grain size through rapid solidification has important consequences with respect to super plastic behaviour of iron-based alloys. It has thus been possible to extend the range of super plasticity in steels to very high carbon contents. The type of super plastic properties achieved through rapid solidification is far superior to those attainable through thermomechanical treatments alone. Ruano et al (1982) have been able to generate ferritic grain sizes of 1.0 to $2.0 \mu \mathrm{m}$ by rapid solidification and achieve tensile elongations in excess of $1400 \mathrm{pct}$ in a white cast iron with $3.0 \%$ $\mathrm{C}$ and $1.5 \% \mathrm{Cr}$. The strain rate sensitivity was high and close to 0.5 . Similarly, Spies and Frommeyer (1991) have been able to achieve tensile elongations of about $175 \%$ at $1323 \mathrm{~K}$ in a tool steel with $3.8 \% \mathrm{C}, 24.9 \% \mathrm{Cr}, 8.7 \% \mathrm{~V}$ and $2.7 \% \mathrm{Mo}$. In this steel, they could achieve ferritic grain sizes below $4 \mu \mathrm{m}$ and carbide particles were dispersed with sizes in the range $1-3 \mu \mathrm{m}$. Frommeyer and Vogt (1981) attribute the high strain 
rate sensitivity of the ultra-high carbon steels to the fine microduplex structure generated by rapid solidification.

\section{Extension of solid solubility}

Increase in solubility of many alloying elements in the base metal has been an important effect of rapid solidification and the underlying causes for such an extension have been reviewed (Ramachandrarao 1988). With respect to steels, the enhancement of solubility opens up the possibility of developing novel precipitation--hardenable steels. Available data on the solubilities in iron before and after rapid solidification are presented in table 2 . In many cases, the enhancement in solid solubility is determined through the measurement of lattice parameter. Figure 4 is an example. Such plots are also used to evaluate the percent change in lattice parameter due to the enhancement of solubility. The change is a measure of the strain introduced through alloying and can be used to estimate the enhancement in strength and hardness as can be seen from figure 5. Table 3 shows the effectiveness of different alloying elements on causing solid solution strengthening. By far, carbon has the most significant effect.

As can be seen, the most spectacular increase occurs with respect to the solubility of boron. The increase in solubility has been exploited by Marko metals to patent a series of steels with superior properties attained through the precipitation of borides (Ray et al 1983; Ray and Isserow 1984). In pure iron, the maximum solubility of boron is only $50 \mathrm{ppm}$ and can be enhanced to several at.\% by rapid solidification. During hot compaction and extrusion of the rapidly solidified material, it precipitates in the form of $\mathrm{M}_{23} \mathrm{~B}_{6}, \mathrm{M}_{2} \mathrm{~B}, \mathrm{MB}_{2}$ or $\mathrm{M}_{5} \mathrm{~B}_{3}$ where $\mathrm{M}$ may be $\mathrm{Cr}, \mathrm{Mo}$, Ti, W etc. In a majority of cases, the borides are either $M_{2} B$ or $M_{23} B_{6}$. The borides precipitate both at the grain boundaries and in the grain (Shrivastava et al 1989). The former are invariably larger in size while the latter can be as fine as $20-30 \mathrm{~nm}$. These boride precipitates enhance yield and tensile strengths considerably with about $10 \%$ increase in ductility at high temperature. Since the borides present at the grain boundaries inhibit grain growth the increment in strength due to grain refinement is retained

Table 2. Limits of solid solubility in rapidly quenched iron-based alloys.

\begin{tabular}{lcc}
\hline & \multicolumn{2}{c}{ Solid solubility limit (at.\%) } \\
\cline { 2 - 3 } Solute & Equilibrium & Extended \\
\hline $\mathrm{B}$ & $0 \cdot 1$ & $4 \cdot 3$ \\
$\mathrm{C}$ & 8.9 & $16 \cdot 2$ \\
$\mathrm{Cu}$ & $7 \cdot 2$ & $15 \cdot 0$ \\
$\mathrm{Ga}$ & $17 \cdot 4$ & 50.0 \\
$\mathrm{Gr}$ & 20.0 & $25 \cdot 0$ \\
$\mathrm{Mo}$ & 26.0 & 40.6 \\
$\mathrm{Rh}$ & $50 \cdot 0$ & 100.0 \\
$\mathrm{Sn}$ & $9 \cdot 8$ & 20.0 \\
$\mathrm{Ti}$ & $9 \cdot 8$ & 16.0 \\
$\mathrm{~W}$ & 13.0 & 20.8 \\
\hline
\end{tabular}




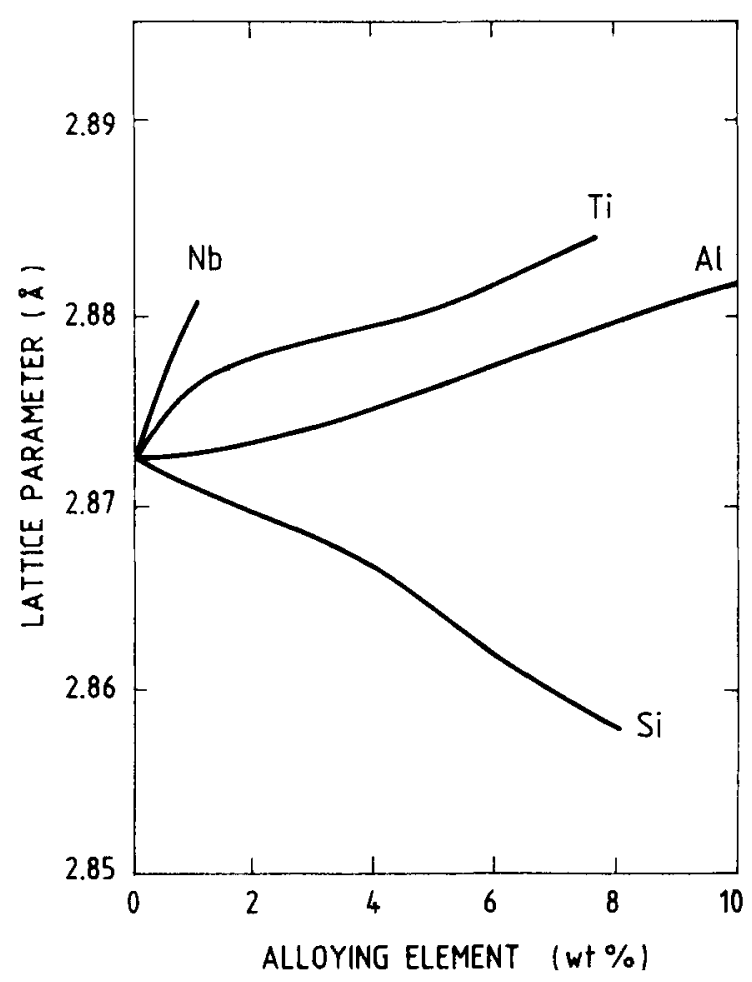

Figure 4. Dependence of lattice parameter of ferrite on concentration of solute element in $17 \%$ Cr steels.
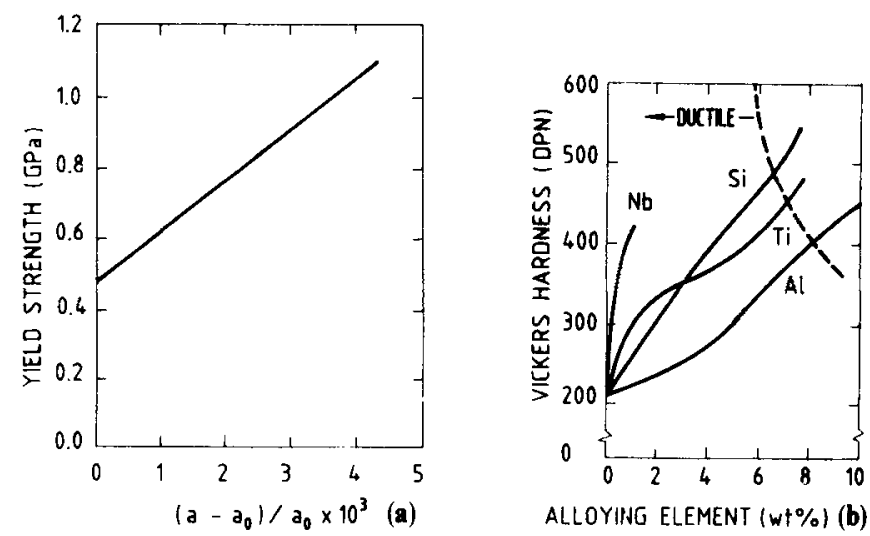

Figure 5. Dependence of (a) yield strength and (b) hardness on the extent of alloying of a Fe- $17 \%$ Cr steel.

even at fairly high temperatures. It also enables the use of conventional powder metallurgy route for consolidation and processing at high temperatures. Solubility extension through rapid solidification is thus proving to be an indispensable method of improving the mechanical properties of iron-based alloys. 
Table 3. Increase in hardness due to solid solution formation in rapidly solidified iron-based alloys.

\begin{tabular}{lcc}
\hline System & Solute & $\begin{array}{c}\text { Increase in hardness }\left(\Delta H_{v} / \text { at. \%) }\right. \\
\text { rapidly solidified }\end{array}$ \\
\hline Fe-C-W & C & 49 \\
Fe-C-Mo & C & 40 \\
Fe-C-Cr & C & 36 \\
Fe-C-W & W & 31 \\
Fe-C-Mo & Mo & 32 \\
Fe-C-Cr & Cr & 9 \\
\hline
\end{tabular}

\section{Metastable phase formation}

Rapid solidification can lead to the formation of metastable crystalline intermediate as well as amorphous phases. We shall mostly confine our attention only to the crystalline phases. Das et al (1989) reviewed the status with respect to the phases obtained by rapid solidification of $\mathrm{Fe}-\mathrm{C}$ and $\mathrm{Fe}-\mathrm{C}-\mathrm{Cr}$ alloys. At the highest rates of cooling attainable, it is possible to produce an amorphous phase and a metastable hexagonal close-packed (hcp) $\varepsilon$-phase in Fe- $(2-5$ wt. $\%) \mathrm{C}$ alloys. This phase can be considered to be solid solution of carbon in metastable hcp iron and has a formula $\mathrm{Fe}_{12} \mathrm{C}_{3}$. The structure bears a relationship with the ordered $\varepsilon$-carbide and decomposes on annealing.

In recent years, a novel use has been found for the metastable phases produced by rapid solidification of $\mathrm{Fe}-\mathrm{C}-\mathrm{M}$ type alloys. In these alloys one can obtain the $\varepsilon$-, $\kappa$-, and $\chi$-phases besides extension of solubility of carbon and $\mathbf{M}$ in austenite and the amorphous phase at certain compositions (Harakawa et al 1985). The $\kappa$ phase has an ordered face-centred cubic structure while the $\chi$ phase has a body-centred cubic structure. The latter of these forms on a fine scale and is brittle by nature. This characteristic has been exploited to produce fine powders of $\mathrm{Fe}-\mathrm{C}-\mathrm{M}$ alloys. It has been demonstrated that the comminuting time for melt-spun tapes can be as low as $15 \mathrm{~min}$ only. It is also possible to reduce sintering times in subsequent processing operations. Even a 60 -min sintering at $1323-1473 \mathrm{~K}$ enables production of a compact with only $10-20 \%$ porosity and an ultrafine dispersion of $M_{7} C_{3}$ and $M_{23} C_{6}$. The resultant hardness is $25-50 \%$ higher than the hardness of conventionally prepared high-carbon alloy steel sintered products. In view of these advantages, the compositions at which the $\chi$ phase forms have been carefully delineated by Harakawa et al (1985). Similar studies along these lines on other commercially important iron-base alloys can help simplify processing techniques and should be pursued vigorously.

\section{Conclusions}

We have briefly reviewed the status with respect to the production, processing and scientific understanding of rapidly solidified materials with specific reference to steels. Abraham (1990) has reviewed the future market for rapidly solidified materials. In general, the market is expected to grow at the rate of $17 \%$ and have a total value of $\$ 290$ million by year 1993. In 1988, iron-base and super alloys had $88 \%$ of the market 
share with the next best being metallic glasses with about $7.6 \%$ of the market. The glassy alloy market is expected to grow and soon may have an equal share as that of iron-base alloys and be about $30 \%$ of the market. It is expected that the first decade of the next century will see the real growth period in the demand for these materials.

\section{References}

Abraham T $1990 \mathrm{~J}$. Metals 426

Adam C M 1986 in Mechanical behaviour of rapidly solidified materials, (eds) S M L Sastry and B A Mac Donald (Warrendale: The Metallurgy Society Inc.) pp 21-40

Anantharaman T R and Ramachandrarao P 1973 The Banaras Metallurgist 537

Aziz M J 1982 J. Phys. 531158

Bae J C and Kelly T F 1988 Scr. Metall. 22691

Cantor B, Kim W T, Bewlay B P and Gillen A G 1991 J. Mater. Sci. 261266

Coriell S R and Sekerka R F 1980 in Rapid solidification processing: principles and technologies, (eds).

R Mehrabian, B H Kear and M Cohen (Baton Rouge: Claitor Publishing Division) pp 35-49

Das N K, Sivaramakrishnan C S and Mohanty O N 1989 Key Engg. Mat. 38 \& 3965

Flemings M C 1974 Solidification processing (New York: McGraw Hill) pp 31-92

Frommeyer G and Vogt E 1981 Steel Res. 58491

Greer A L 1988 Mater. Sci. Engg. 97285

Greer A L 1991 Mater. Sci. Engg. A133 16

Harakawa Y. Inoue A and Masumoto T 1985 Sci. Rep. (Tohoku University) 32277

Hayzelden C. Rayment J J and Cantor B 1983 Acta Metall. 31379

Inokutı Y and Cantor B 1977 J. Mater. Sci. 12946

Inokuti $\mathrm{Y}$ and Cantor B 1982 Acta Metall. 30343

Jones H 1977 in Vacancies 76 leds) R E Smallman and J E Harris (London: Metals Society) p. 175

Kim W T and Cantor B 1990 Scr. Metall. 24633

Lawley A $1985 \mathrm{~J}$. Metals 3715

Ramachandrarao P $1988 \mathrm{~J}$. Sci. Res. (Banaras Hindu University) 3827

Ramachandrarao P, Scott M G and Chadwick G A 1972 Philos. Mag. 25961

Ray R and Isserow S 1984 in Proc. of Conf. on powder metallurgy in defence technology, (New Jersey: Metal Powder Industries Federation) 6 pp 99-116

Ray R. Panchananthan V and Isserow S 1983 J. Metals 3531

Ruano O A. Eiselstein L E and Sherby O D 1982 Met. Trans. A13 1785

Shrivastava P K. Tiwari A N and Gopinathan V 1989 Key Engg. Mat. 38 \& 39143

Spies H J and Frommeyer G 1991 Mater. Sci. Tech. 7718

Staski W, Chandhok V K and Pinnow K E 1988 Met. Pow. Rep. 43409

Wood J V and Honeycombe R W K 1974 J. Mater. Sci. 91183 